\title{
Expression of Matrix Metalloproteinases in Intraductal Papillary Mucinous Neoplasm of the Pancreas
}

\author{
MASANORI AKASHI ${ }^{1}$, TORU HISAKA ${ }^{1}$, HISAMUNE SAKAI $^{1}$, HIROTO ISHIKAWA $^{1}$, \\ RYUICHI KAWAHARA ${ }^{1}$, YUICHI GOTO ${ }^{1}$, YORIKO NOMURA ${ }^{1}$, MASAFUMI YASUNAGA ${ }^{1}$, \\ FUMIHIKO FUJITA ${ }^{1}$, MASAHIKO TANIGAWA ${ }^{2}$, YOSHIKI NAITO ${ }^{2}$, JUN AKIBA ${ }^{2}$, \\ HIROHISA YANO $^{3}$, HIROYUKI TANAKA ${ }^{1}$, YOSHITO AKAGI ${ }^{1}$ and KOJI OKUDA ${ }^{1}$ \\ Departments of ${ }^{1}$ Surgery and ${ }^{3}$ Pathology, Faculty of Medicine, Kurume University, Kurume, Japan; \\ ${ }^{2}$ Department of Diagnostic Pathology, Kurume University Hospital, Kurume, Japan
}

\begin{abstract}
Background/Aim: Intraductal papillary mucinous neoplasm (IPMN) has a variety of histological and morphological appearances. Matrix metalloproteinases (MMPs) have been considered to be associated with tumor progression or poor prognosis. The aim of this study was to elucidate the molecular basis of IPMN variation in different types of lesions. Materials and Methods: The expression of MMP-1,2,7,9 in 51 cases of IPMN were investigated. The MMP score was calculated as the sum of the score of staining distribution and the score of the intensity staining. Results: MMP scores were correlated with histological grade, histological subtype, and type of invasion. MMP high expression groups (MMP score $\geq 5)$ had worse prognosis than low-expression groups. Conclusion: MMP expression varied between different types of IPMN, a result supporting differences in molecular basis of malignancies. These considerations may be helpful for optimal management or treatment according to various types of IPMN.
\end{abstract}

Intraductal papillary mucinous neoplasm (IPMN) of the pancreas is characterized by mucus production, cystic dilation of the pancreatic duct, and papillary proliferation of pancreatic intraductal epithelium (1). Histologically, IPMN includes variable grades of dysplasia. When IPMN is diagnosed at the stage of low-grade to high-grade dysplasia, its treatment by resection offers an extremely good prognosis; however, once it

This article is freely accessible online.

Correspondence to: Toru Hisaka, MD, Ph.D., Department of Surgery, Kurume University Faculty of Medicine, 67 Asahi-machi, Kurume, Fukuoka 830-0011, Japan. Tel: +81 942317566, Fax: +81 942340709, e-mail: thisaka@med.kurume-u.ac.jp

Key Words: Intraductal papillary mucinous neoplasm, histological subtype, histological grade, type of invasion, matrix metalloproteinases, immunohistochemistry. has progressed to IPMN-derived invasive carcinoma (IPMC), its prognosis is poor $(2,3)$. The classifications according to epithelial morphology and mucus traits have recently been reported to have clinicopathological significance $(4,5)$. Thus, based on its epithelial morphology and mucus traits, IPMN can be classified as either gastric type, intestinal type, pancreatobiliary type, or oncocytic type, and these four subtypes have different clinicopathological characteristics. IPMC is classified as tubular-type or colloid-type depending on the morphology of invasion, and these types have been found to be associated with different prognoses (5). These findings suggest the existence of molecular differences associated with the growth and invasion of each type of IPMN.

Matrix metalloproteinases (MMPs) are enzymes that specifically degrade structural constituents of the extracellular matrix. They have been implicated in the processing and activation of physiologically active substances, maintenance, repair, and regeneration of tissue architecture, and in tumour invasion, metastasis, growth, progression, and neovascularisation $(6,7)$. In normal tissues, the levels of MMP expression and activity/inhibition is maintained; however, in tumour tissues this equilibrium is disrupted, causing overexpression of multiple MMPs (7). Studies have identified a correlation between MMP overexpression and malignancy in several types of cancer tissues (8-11), and a number of reports have found that MMP expression is also associated with invasion, metastasis, and poor prognosis in pancreatic cancer (12-14). The small number of studies on MMP expression in IPMN include those that suggest MMP involvement in the progression and invasion of IPMN $(15,16)$. However, no study has addressed differences in MMP expression between the different histological subtypes of IPMN or different types of invasion. In this study, we compared the levels of expression of MMP1, MMP2, MMP7, and MMP9 in IPMN to investigate the relationship between MMP expression and IPMN and identify the molecular signature of the differences between IPMN types and subtypes. 
Table I. Antibodies for immunohistochemistry.

\begin{tabular}{lccccc}
\hline & Antibody name & Manufacturer & Host animal/Clonality & Pretreatment & Dilution \\
\hline MMP1 & Collagenase-1 Ab-6 & Thermo scientific & Rabbit/polyclonal & Autoclave $121^{\circ} \mathrm{C} 10 \mathrm{~min}$ & $1: 1$ \\
MMP2 & MMP2 Antibody & Novus biologicals & Rabbit/polyclonal & None & None \\
MMP7 & MMP7 Antibody & Gene Tex & Rabbit/polyclonal & Autoclave $121^{\circ} \mathrm{C} 10 \mathrm{~min}$ & $1: 25$ \\
MMP9 & 92kDa Collagenase IV & Thermo scientific & Rabbit/polyclonal & 1:2 \\
\hline
\end{tabular}

\section{Materials and Methods}

Patients and classifications of IPMN. We investigated 51 IPMN lesions resected from 51 patients in Kurume University Hospital or associated hospitals between 1996 and 2012, and compared these with 30 pancreatic ductal adenocarcinoma (PDAC) lesions resected from 30 patients between 2006 and 2009. Histological grade was classified as low-grade-intermediate dysplasia, high-grade dysplasia, or IPMC, by WHO classification in 2010 (17). Histological subtypes were classified as either gastric type, intestinal type, pancreatobiliary type, or oncocytic type on the basis of epithelial morphology as evidenced by haematoxylin-eosin (HE) staining and MUC staining (4), and the morphology of invasion as tubular or colloid type.

Immunohistochemical staining. Table I shows the names, suppliers, clones, and dilutions of the primary antibodies used. Formalin-fixed, paraffin-embedded slices of each lesion were deparaffinized, and those slices used for MMP1 or MMP9 immunohistochemical staining were subjected to antigen unmasking by autoclaving at $121^{\circ} \mathrm{C}$ for $10 \mathrm{~min}$ in $0.01-\mathrm{M}$ citric acid buffer solution. Endogenous peroxidase blocking was carried out with $3 \%$ hydrogen peroxide at room temperature for $10 \mathrm{~min}$. The samples were incubated with primary antibodies at room temperature for $30 \mathrm{~min}(90 \mathrm{~min}$ for MMP7 immunohistochemical staining). Samples were then incubated with peroxidase-conjugated secondary antibodies coupled with polymer dextran (EnVision-HRP, DAKO, Santa Clara, CA, USA) at room temperature for $30 \mathrm{~min}$, after which the stain was developed with 3, 3'-diaminobenzidine (DAB) and counterstaining with haematoxylin was carried out.

Evaluation of MMP staining results. The most atypical area of each lesion was observed. Following the methods of Shimizu et al. (18), both the distribution (the percentage of epithelial cells stained) and the intensity of staining were assessed semi quantitatively. The following system was used to score the distribution of epithelial cells stained: none $(0 \%)=0$, focal $(1 \%-30 \%)=1$, multifocal $(31 \%$ $70 \%)=2$, or diffuse $(71 \%-100 \%)=3$. The intensity of staining was graded as follows: none $=0$, weak $=1$, moderate $=2$, or strong $=3$. The MMP score was calculated as the sum of these two scores. Staining was evaluated by three investigators. The MMP scores of resected PDAC and IPMN lesions were compared, and the scores were also compared for the different macroscopic types, histological grades, histological subtypes, and types of invasion of IPMN. MMP scores $\geq 5$ were classified as high expression and $\leq 4$ as low expression, and overall survival was compared for these two groups.

Statistical analysis. A Kruskal-Wallis test was used to compare scores between different groups. For the groups classified by MMP
Table II. Classification and proportion of 51 IPMNS.

\begin{tabular}{lrrrrrr}
\hline & Total & \multicolumn{5}{c}{ Histological subtype } \\
\cline { 2 - 7 } & & G & I & PB & O & Unclassified \\
\cline { 2 - 7 } & 51 & 18 & 22 & 8 & 1 & 2 \\
\hline Histological grade & & & & & & \\
LID & 16 & 14 & 2 & 0 & 0 & 0 \\
HGD & 11 & 3 & 7 & 0 & 0 & 1 \\
IPMC & 24 & 1 & 13 & 8 & 1 & 1 \\
Type of invasion & & & & & & \\
TUB & 17 & 1 & 6 & 8 & 1 & 1 \\
COL & 7 & 0 & 7 & 0 & 0 & 0 \\
\hline
\end{tabular}

G: Gastric type; I: intestinal type; PB: pancreatobiliary type; O: oncocytic type; LID: low grade-intermediate dysplasia; HGD: highgrade dysplasia; IPMC: IPMN-derived invasive carcinoma; TUB: tubular type; COL: colloid type.

score, Kaplan-Meier curves were generated for overall survival in both groups, and assessed using a log-rank test. Analysis was carried out using JMP Pro11.0.0 (SAS Institute Inc., Cary, NC, USA), with $p<0.05$ regarded as significant.

\section{Results}

Table II shows the 51 IPMN lesions and their classifications. Nearly all the gastric type lesions were non-invasive IPMN. Nine of the intestinal type lesions were non-invasive IPMN and 13 were IPMC. All the pancreatobiliary type lesions were IPMC. The types of invasion of IPMC lesions were tubular type in 17 cases and colloid type in seven patients. The tubular type lesions comprised 8 pancreatobiliary, 6 intestinal, 1 gastric, 1 oncocytic, and 1 unclassifiable type. All the colloid type lesions were intestinal type.

MMPs were expressed mainly in the cytoplasm of tumor cells in both IPMN and PDAC (Figure 1). There was almost no expression in normal pancreatic duct epithelium.

Table III shows the comparison of mean MMP scores between groups. A comparison of PDAC and IPMN showed that all MMP scores other than MMP9 were significantly higher in PDAC tissues. In terms of histological grades, 

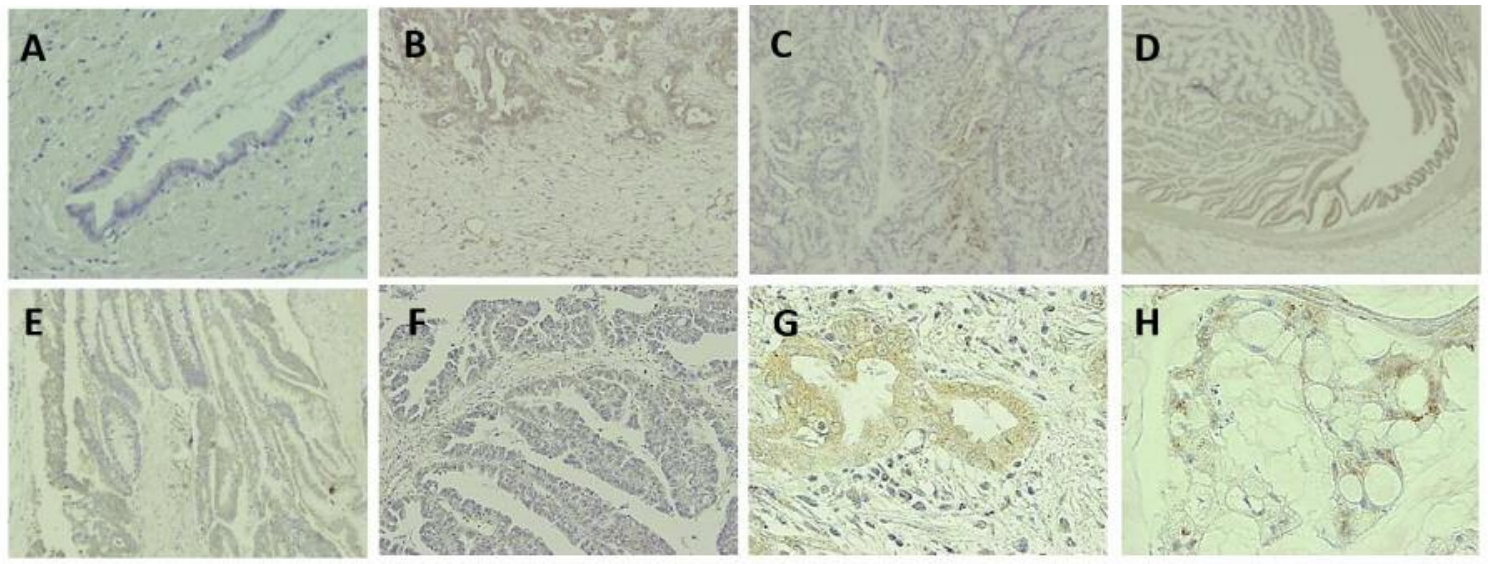

Figure 1. Representative results of MMP immunohistochemistry. (A) Normal pancreatic duct $(\times 100)$, (B) pancreatic ductal adenocarcinoma $(\times 100)$, (C) gastric type IPMN (x100), (D) intestinal type IPMN (×100), (E) pancreatobiliary type IPMN $(\times 100),(F)$ oncocytic type IPMN $(\times 100),(G)$ tubular type IPMN-derived invasive carcinoma $(\times 200),(H)$ colloid type IPMN-derived invasive carcinoma $(\times 200)$.

Table III. Comparison of mean MMP scores in each group.

\begin{tabular}{|c|c|c|c|c|c|c|c|c|c|}
\hline & & \multicolumn{2}{|c|}{ MMP1 } & \multicolumn{2}{|c|}{ MMP2 } & \multicolumn{2}{|c|}{ MMP7 } & \multicolumn{2}{|c|}{ MMP9 } \\
\hline & & Mean scores & $p$-Value & Mean scores & $p$-Value & Mean scores & $p$-Value & Mean scores & $p$-Value \\
\hline \multirow[t]{2}{*}{ IPMN vs. PDAC } & PDAC $(n=30)$ & 4.53 & $p=0.036$ & 4.7 & $p=0.0468$ & 5.33 & $p<0.0001$ & 4.57 & $\mathrm{~ns}$ \\
\hline & IPMN $(n=51)$ & 3.49 & & 3.69 & & 3.55 & & 4.22 & \\
\hline \multirow[t]{3}{*}{ Histological grade } & LID $(n=16)$ & 2.37 & $p=0.0057$ & 2.44 & $p=0.0171$ & 2.56 & $p=0.0080$ & 2.69 & $p<0.0001$ \\
\hline & $\operatorname{HGD}(\mathrm{n}=11)$ & 3.82 & & 3.82 & & 3.55 & & 4.73 & \\
\hline & IPMC $(n=24)$ & 4.08 & & 4.46 & & 4.21 & & 4.96 & \\
\hline \multirow[t]{3}{*}{ Histological subtype } & $\mathrm{G}(\mathrm{n}=18)$ & 2.33 & $p=0.0002$ & 2.67 & $p=0.0010$ & 2.83 & $p=0.0104$ & 3.06 & $p=0.0012$ \\
\hline & $I(n=22)$ & 3.68 & & 3.55 & & 3.41 & & 4.68 & \\
\hline & $\mathrm{PB}(\mathrm{n}=8)$ & 5 & & 5.75 & & 4.88 & & 5.13 & \\
\hline \multirow[t]{2}{*}{ Non-invasive IPMN } & $G(n=17)$ & 2.35 & $p=0.0034$ & 2.59 & $\mathrm{~ns}$ & 2.71 & $\mathrm{~ns}$ & 2.94 & $p=0.0127$ \\
\hline & I $n=9)$ & 4 & & 3.67 & & 3.33 & & 4.56 & \\
\hline \multirow[t]{2}{*}{ IPMC } & $\mathrm{I}(\mathrm{n}=13)$ & 3.46 & $p=0.0292$ & 3.46 & $p=0.0057$ & 3.46 & $p=0.0366$ & 4.77 & ns \\
\hline & $\mathrm{PB}(\mathrm{n}=8)$ & 5 & & 5.75 & & 4.88 & & 5.13 & \\
\hline \multirow{2}{*}{ Type of invasion } & TUB $(n=17)$ & 4.82 & $p=0.001$ & 5.41 & $p=0.0003$ & 4.94 & $p=0.0003$ & 5.12 & $\mathrm{~ns}$ \\
\hline & $\operatorname{COL}(\mathrm{n}=7)$ & 2.29 & & 2.14 & & 2.43 & & 4.71 & \\
\hline
\end{tabular}

PDAC: Pancreatic ductal adenocarcinoma; LID: low grade-intermediate dysplasia; HGD: high-grade dysplasia; IPMC: IPMN-derived invasive carcinoma; G: gastric type; I: intestinal type; PB: pancreatobiliary type; TUB: tubular type; COL: colloid type; ns, not significant.

MMP scores were highest in IPMC, followed by high-grade dysplasia, and low-grade-intermediate dysplasia, and these differences were statistically significant. A comparison of histological subtypes found that MMP scores were highest for pancreatobiliary type, followed by intestinal and then gastric type, and these differences were statistically significant (oncocytic and unclassifiable types were excluded because their numbers were too small). We further divided histological subtypes into non-invasive IPMN and IPMC and compared their MMP scores. The MMP scores of gastric and intestinal types, which accounted for the majority of noninvasive IPMN were compared. Intestinal type tended to have higher MMP scores than gastric type, and this difference was statistically significant for MMP1 and MMP9. We then compared the MMP scores of intestinal and pancreatobiliary types, which accounted for the majority of IPMC. Pancreatobiliary type tended to have higher MMP scores than intestinal type, and this difference was statistically significant for MMP1, MMP2, and MMP7. A comparison of the type of invasion showed that all MMP scores other than MMP9 were significantly higher in tubular type than in colloid type.

Lesions were divided into a high expression group with an MMP score $\geq 5$ and a low expression group with an MMP 

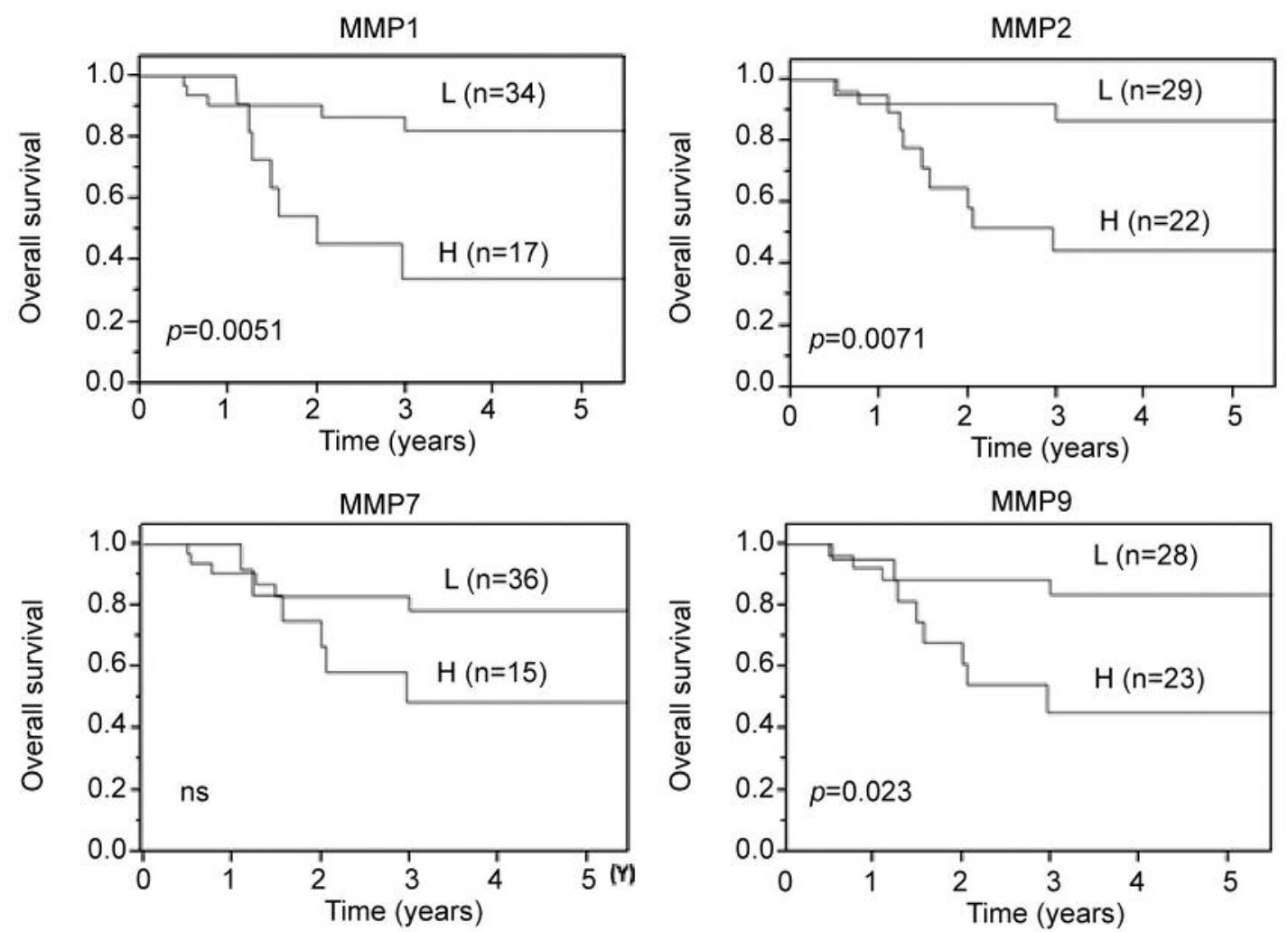

Figure 2. Overall survival between high MMP expression group and low MMP expression group. L: Low MMP expression group (MMP score $\leq 4$ ), H: high MMP expression group (MMP score $\geq 5)$. High MMP expression groups had worse prognosis than low MMP expression groups. There were significant differences regarding MMP1, 2 and 9 expression.

score $\leq 4$, and overall survival between the two groups was compared. The high expression group tended to have a poorer prognosis than the low-expression group, and this difference was statistically significant for MMP1, MMP2, and MMP9 (Figure 2).

\section{Discussion}

This study was focused on the involvement of MMPs in tumor progression, and MMP expression in IPMN was assessed. We compared histologically and morphologically the expression of MMP1, MMP2, MMP7, and MMP9, well known as secreted MMPs, and found that MMP expression varied in lesions with different histological grade, histological subtype, and type of invasion.

MMPs are reportedly more strongly expressed in pancreatic carcinoma tissue than in normal tissue, and in most cases the levels of expression are associated with malignancy $(13,14)$. The scores for MMP1, MMP2, and MMP7 were significantly higher in PDAC tissues compared with that of IPMN. Few studies have compared MMP expression in PDAC and IPMN, but there have been reports that MMP expression is more intense in PDAC than in IPMN $(15,16)$, and our own results support this. These results suggest that differences in MMP expression may underlie differences in these malignancies.

Our comparison between IPMN lesions of different histological grade found that MMP expression increased depending on the degree of dysplasia, with a significant difference for all MMP scores. Nishikawa et al. have reported that MMP7 expression levels varied between noninvasive IPMN and invasive IPMC, and hypothesized that MMP7 may contribute to invasion (15). Tamahashi et al. have identified a correlation between the rate of MMP7 expression and histological grade of IPMN, and suggested that it may be involved in the progression from adenoma to carcinoma. They have also suggested that MMP1, MMP2, and MMP9 may be involved in tumor progression process in the early stages of IPMN (16). Studies on colorectal and oesophageal carcinoma have also found that MMPs are expressed in the initial stage of tumour growth, and they are involved in tumorigenesis and progression $(19,20)$. In this study, we found that there was almost no MMP expression in normal pancreatic duct epithelium, but expression began to appear in low-grade dysplasia, the first stage of tumor development, and the level of expression increased according to histological grade, suggesting that MMPs may be involved in tumorigenesis and progression of IPMN. 
Clinicohistopathological differences between the histological subtypes of IPMN have been reported (3-5), and their characteristics are broadly understood. However, few studies have addressed differences at the molecular level between subtypes. In this study, MMP scores varied significantly between different subtypes. A comparison between subtypes matched by degree of dysplasia also showed significant differences in several MMP scores. This suggests that the different histological subtypes of IPMN may have different molecular bases.

In this study, we found that tubular type lesions had significantly higher MMP scores for MMP1, MMP2, and MMP7 compared with that of colloid type lesions. These types of invasion may contribute to the difference in prognosis between patients with pancreatobiliary and intestinal type IPMN, because all the pancreatobiliary type IPMC lesions had tubular type invasive morphology, whereas over half of intestinal type IPMC lesions had colloid type invasive morphology.

Previous studies have reported that MMP expression is associated with poor prognosis for patients with a wide variety of tumours (8-11). In this study, overall survival curves found that the prognosis tended to be poor for those in the high expression groups (MMP score $\geq 5$ ) for all MMPs, and that this difference was significant for MMP1, MMP2, and MMP9. These findings suggested that the evaluation of MMP expression in IPMN may both be helpful for predicting prognosis and for providing a marker for malignancy.

However, the regulatory mechanisms of MMPs are complex and there have been few functional studies of MMPs in IPMN. It remains unknown which specific genes are associated with MMP overexpression in IPMN. Further studies are, therefore, required.

\section{Conclusion}

In the present study, MMP expression varied between different histological grade, macroscopic type, histological subtype, and type of invasion of IPMN, a result supporting differences in the molecular level of these malignancies. Furthermore, MMPs may be involved in the development, progression and prognosis of IPMN. Therefore, it was suggested that these evaluations may be helpful for optimal management or treatment according to various types of IPMN.

\section{Conflicts of Interest}

The Authors declare no conflicts of interest regarding this study.

\section{Authors' Contributions}

Masanori Akashi designed the study and wrote the draft of the manuscript. Masanori Akashi, Toru Hisaka, Yoshiki Naito conducted the immunohistochemical analysis. Toru Hisaka contributed to analysis and interpretation of data, and assisted in the preparation of the manuscript. All other Authors have contributed to data collection and interpretation, and critically reviewed the manuscript.

\section{References}

1 Tanaka M, Kobayashi K, Mizumoto $\mathrm{K}$ and Yamaguchi $\mathrm{K}$ : Clinical aspects of intraductal papillary mucinous neoplasm of the pancreas. J Gastroenterol 40: 669-675, 2005. PMID: 16082582. DOI: $10.1007 / \mathrm{s} 00535-005-1646-4$

2 Moris D, Damaskos C, Spartalis E, Papalampros A, Vernadakis S, Dimitroulis D, Griniatsos J, Felekouras E and Nikiteas N: Updates and critical evaluation on novel biomarkers for the malignant progression of intraductal papillary mucinous neoplasms of the pancreas. Anticancer Res 37: 2185-2194, 2017. PMID: 28476781. DOI: 10.21873/anticanres.11553

3 Hisaka T, Horiuchi $\mathrm{H}$, Uchida S, Ishikawa H, Kawahara R, Kawashima Y, Akashi M, Mikagi K, Ishida Y, Okabe Y, Nakayama M, Naito Y, Yano H, Taira T, Kawahara A, Kage M, Kinoshita H and Shirozu K: Potential usefulness of mucin immunohistochemical staining of preoperative pancreatic biopsy or juice cytology specimens in the determination of treatment strategies for intraductal papillary mucinous neoplasm. Oncol Rep 30: 20352041, 2013. PMID: 24008495 . DOI: $10.3892 /$ or.2013.2720

4 Furukawa T, Hatori T, Fujita I, Yamamoto M, Kobayashi M, Ohike N, Morohoshi T, Egawa S, Unno M, Takao S, Osako M, Yonezawa S, Mino-Kenudson M, Lauwers GY, Yamaguchi H, Ban S and Shimizu M: Prognostic relevance of morphological types of intraductal papillary mucinous neoplasms of the pancreas. Gut 60: 509-516, 2011. PMID: 21193453. DOI: 10.1136/gut.2010.210567

5 Mino-Kenudson M, Fernández-del Castillo C, Baba Y, Valsangkar NP, Liss AS, Hsu M, Correa-Gallego C, Ingkakul T, Perez Johnston R, Turner BG, Androutsopoulos V, Deshpande V, McGrath D, Sahani DV, Brugge WR, Ogino S, Pitman MB, Warshaw AL and Thayer SP: Prognosis of invasive intraductal papillary mucinous neoplasm depends on histological and precursor epithelial subtypes. Gut 60: 1712-1720, 2011. PMID: 21508421. DOI: $10.1136 /$ gut.2010.232272

6 Nagase H and Woessner JF Jr.: Matrix metalloproteinases. J Biol Chem 274: 21491-21494, 1999. PMID: 10419448. DOI: 10.1074/jbc.274.31.21491

7 Egeblad $\mathrm{M}$ and Werb $\mathrm{Z}$ : New functions for the matrix metalloproteinases in cancer progression. Nat Rev Cancer 2: 161-174, 2002. PMID: 11990853. DOI: $10.1038 / \mathrm{nrc} 745$

8 Zhang M, Zhu GY, Gao HY, Zhao SP and Xue Y: Expression of tissue levels of matrix metalloproteinases and tissue inhibitors of metalloproteinases in gastric adenocarcinoma. J Surg Oncol 103: 243-247, 2011. PMID: 21337552. DOI: 10.1002/jso.21824

9 Greco M, Arcidiacono B, Chiefari E, Vitagliano T, Ciriaco AG, Brunetti FS, Cuda G and Brunetti A: HMGA1 and MMP-11 are overexpressed in human non-melanoma skin cancer.Anticancer Res 38: 771-778, 2018. PMID: 29374701. DOI: 10.21873/ anticanres. 12283

$10 \mathrm{Gu}$ ZD, Li JY, Li M, Gu J, Shi XT, Ke Y and Chen KN: Matrix metalloproteinases expression correlates with survival in patients with esophageal squamous cell carcinoma. Am J Gastroenterol 100: 1835-1843, 2005. PMID: 16086722. DOI: 10.1111/j.15720241.2005.50018.x 
11 Köhrmann A, Kammerer U, Kapp M, Dietl J and Anacker J: Expression of matrix metalloproteinases (MMPs) in primary human breast cancer and breast cancer cell lines: New findings and review of the literature. BMC Cancer 9: 188, 2009. PMID: 19531263. DOI: 10.1186/1471-2407-9-188

12 Nagakawa Y, Aoki T, Kasuya K, Tsuchida A and Koyanagi Y:Histologic features of venous invasion, expression of vascular endothelial growth factor and matrix metalloproteinase- 2 and matrix metalloproteinase-9, and the relation with liver metastasis in pancreatic cancer.Pancreas 24: 169-178, 2002. PMID: 11854622. DOI: 10.1097/00006676-200203000-00008

13 Yamamoto H, Itoh F, Iku S, Adachi Y, Fukushima H, Sasaki S, Mukaiya $\mathrm{M}$, Hirata $\mathrm{K}$ and Imai $\mathrm{K}$ : Expression of matrix metalloproteinases and tissue inhibitors of metalloproteinases in human pancreatic adenocarcinomas: clinicopathologic and prognostic significance of matrilysin expression. J Clin Oncol 19: 1118-1127, 2001. PMID: 11181677. DOI: 10.1200/JCO. 2001.19.4.1118

14 Jones LE, Humphreys MJ, Campbell F, Neoptolemos JP and Boyd MT: Comprehensive analysis of matrix metalloproteinase and tissue inhibitor expression in pancreatic cancer: increased expression of matrix metalloproteinase-7 predicts poor survival. Clin Cancer Res 10: 2832-2845, 2004. PMID: 15102692. DOI: 10.1158/1078-0432.CCR-1157-03

15 Nishikawa N, Kimura Y, Okita K, Zembutsu H, Furuhata T, Katsuramaki T, Kimura S, Asanuma $\mathrm{H}$ and Hirata K: Intraductal papillary mucinous neoplasms of the pancreas: an analysis of protein expression and clinical features. J Hepatobiliary Pancreat Surg 13: 327-335, 2006. PMID: 16858545. DOI: 10.1007/ s00534-005-1073-1
16 Tamahashi U, Kumagai J, Takizawa T, Sekine M and Eishi Y: Expression and intracellular localization of matrix metalloproteinases in intraductal papillary mucinous neoplasms of the pancreas. Virchows Arch 453: 79-87, 2008. PMID: 18500535. DOI: 10.1007/s00428-008-0617-6

17 Adsay NV, Fukushima N, Furukawa T, Hruban RH, Klimstra DS, Klöppel G, Offerhaus GJA, Pitman MB, Shimizu M and Zamboni G: Intraductal neoplasms of the pancreas. In: Bosman FT, Carneiro F, Hruban RH, and Theise ND, (eds.). WHO Classification of Tumours of the Digestive System (4th edition). Lyon, France. IARC Press: pp. 304-313, 2010.

18 Shimizu M, Saitoh Y and Itoh H: Immunohistochemical staining of Ha-ras oncogene product in normal, benign, and malignant human pancreatic tissues. Hum Pathol 21: 607-612, 1990. PMID: 2161789. DOI: 10.1016/S0046-8177(96)90006-4

19 Jeffery N, McLean MH, El-Omar EM and Murray GI: The matrix metalloproteinase/tissue inhibitor of matrix metalloproteinase profile in colorectal polyp cancers. Histopathology 54: 820-828, 2009. PMID: 19635101. DOI: 10.1111/j.13652559.2009.03301.x

20 Samantaray S, Sharma R, Chattopadhyaya TK, Gupta SD and Ralhan R: Increased expression of MMP-2 and MMP-9 in esophageal squamous cell carcinoma. J Cancer Res Clin Oncol 130: 37-44, 2004. PMID: 14569466. DOI: 10.1007/s00432-0030500-4
Received May 23, 2019

Revised June 20, 2019

Accepted June 21, 2019 